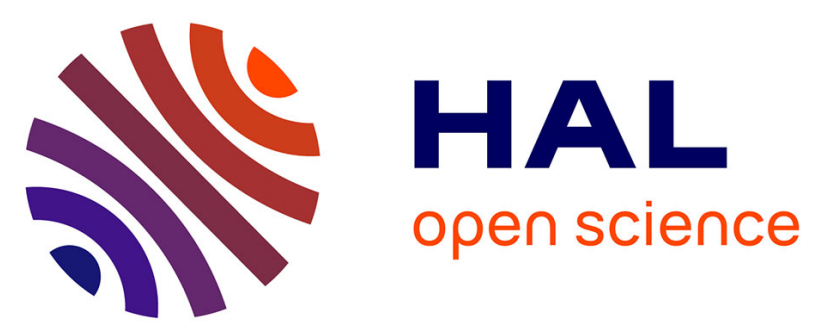

\title{
Simple and efficient polyoxomolybdate-mediated synthesis of novel graphene and metal nanohybrids for versatile applications
}

Shuangshuang Zhang, Rongji Liu, Shiwen Li, Anne Dolbecq, Pierre Mialane, Lin Suo, Lihua Bi, Baofang Zhang, Tianbo Liu, Caixia Wu, et al.

\section{To cite this version:}

Shuangshuang Zhang, Rongji Liu, Shiwen Li, Anne Dolbecq, Pierre Mialane, et al.. Simple and efficient polyoxomolybdate-mediated synthesis of novel graphene and metal nanohybrids for versatile applications. Journal of Colloid and Interface Science, 2018, 514, pp.507-516. 10.1016/j.jcis.2017.12.039 . hal-02335660

\section{HAL Id: hal-02335660 \\ https://hal.science/hal-02335660}

Submitted on 28 Oct 2019

HAL is a multi-disciplinary open access archive for the deposit and dissemination of scientific research documents, whether they are published or not. The documents may come from teaching and research institutions in France or abroad, or from public or private research centers.
L'archive ouverte pluridisciplinaire HAL, est destinée au dépôt et à la diffusion de documents scientifiques de niveau recherche, publiés ou non, émanant des établissements d'enseignement et de recherche français ou étrangers, des laboratoires publics ou privés. 


\section{Simple and efficient polyoxomolybdate-mediated syn- thesis of novel graphene and metal nanohybrids for versatile applications}

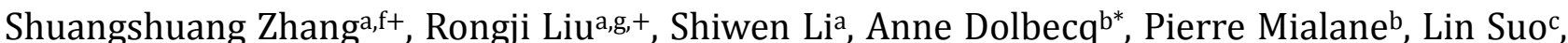
Lihua Bic*, Baofang Zhangd, Tianbo Liud, Caixia Wue, Likai Yane*, Zhongmin Sue, Guangjin Zhanga* $^{a^{*}}$ and Bineta Keitah

\footnotetext{
${ }^{a}$ Key Laboratory of Green Process Engineering, Institute of Process Engineering, Chinese Academy of Sciences, 100190, Beijing, China.

${ }^{b}$ Institut Lavoisier de Versailles, UVSQ, CNRS, Université Paris-Saclay, Versailles, France. ${ }^{c}$ College of Chemistry, Jilin University, Changchun 130012, P. R. China.

${ }^{d}$ Department of Polymer Science, University of Akron, Akron, OH 44325-3909.

${ }^{e}$ Faculty of Chemistry, Northeast Normal University, 130024, Changchun, China.

${ }^{f}$ College of Chemistry, Chemical Engineering and Materials Science, Collaborative Innovation Center of Functionalized Probes for Chemical Imaging in Universities of Shandong, Key Laboratory of Molecular and Nano Probes, Ministry of Education, Institute of Molecular and Nano Science, Shandong Normal University, Jinan 250014, P. R. China.

${ }^{g}$ Institute of Inorganic Chemistry I, Ulm University, Albert-Einstein-Allee 11, 89081, Ulm, Germany.

${ }^{h}$ Retired
}

\begin{abstract}
The application of nanohybrids based on
\end{abstract} polyoxomolybdates, reduced graphene oxide (rGO) and/or metal nanoparticles (NPs) high-performance electrode materials in electrocatalysis and energy storage devices is promising but still limited due to the complexity and the cost of the synthesis. Here we introduce a simple polyoxomolybdate, $\left[\mathrm{MoV}_{4} \mathrm{O}_{8}(\mathrm{OH})_{2}\left(\mathrm{H}_{2} \mathrm{O}\right)_{2}\left(\mathrm{C}_{4} \mathrm{O}_{4}\right)_{2}\right]^{2-}(\mathrm{MoS})$, as reducing and stabilizing agent for the facile and one-pot syntheses of large quantity of highly stable MoS/rGO and MoS/Au NPs nanohybrids in aqueous solution without any catalyst or toxic co-solvent. They were characterized by various physical techniques and electrochemistry which confirm strong interaction between MoS and rGO sheets. We also used DFT calculations to investigate the affinity between MoS or its neutral form with graphene. The adsorption energy for the most stable configuration is $-1.97 \mathrm{eV}$, indicating a strong adsorption process of MoS, which can also be confirmed by the distance $(3.04 \AA)$ and the charge transfer $(0.86$ e) between MoS and graphene. These observations are also consistent with the electrochemical results which underscore the excellent redox properties and high stability of MoS/rGO. Importantly, the MoS/rGO nanohybrids are excellent noble metal-free electrocatalysts for hydrogen peroxide reduction with high sensitivity, large detection range and low detection limit. Finally, the preliminary tests reveal that the electrode materials based on MoS/rGO and a low-cost carbon cloth (CC) composite MoS/rGO/CC may have a potential for an application in energy storage as performant and flexible supercapacitor, showing specific capacitance as high as $870 \mathrm{~F} \mathrm{~g}^{-1}$ at $10 \mathrm{mV} \mathrm{s}^{-1}$ and excellent stability after 5,000 cycles.

Keywords: multifunction materials, reduced graphene 
oxide, polyoxomolybdate, sensor for hydrogen peroxide, flexible supercapacitor electrodes.

\section{Introduction}

Recently, nanohybrid materials based on polyoxometalates (POMs), reduced graphene oxide (rGO) and/or metal (Ag, $\mathrm{Au}, \mathrm{Pd}$ or $\mathrm{Pt}$ ) nano-objects have attracted considerable attention owing to their promising applications in various fields such as photo-electro-catalysis, energy conversion and storage, molecular sensors and electronics [1-8]. POMs are a vast class of early transition metal-oxygen clusters built from the connection of $\left\{\mathrm{MO}_{\mathrm{x}}\right\}$ polyhedra, $\mathrm{M}$ being an early d-block element in high oxidation state, usually $\mathrm{V}^{\mathrm{IV}, \mathrm{V}}, \mathrm{Mo}^{\mathrm{VI}}$ or $\mathrm{W}^{\mathrm{VI}}$ and characterised by a broad diversity of physical and chemical properties [9-25]. A large variety of POMs undergo fast and chemically reversible multi-electron processes without decomposition and thus can act as effective electron transfer catalysts in the homogeneous liquid phase [20,24]. Due to the low electrical conductivity and the high solubility of many POMs in various media, a prerequisite for their applications as effective heterogeneous electrocatalysts is that they must be appropriately immobilised onto highly conductive supports with large specific surface areas, such as rGO and metal nanoparticles (NPs). POM/metal NPs, POM/rGO and metal NPs/POM/rGO nanohybrids were fabricated by using various methods such as high-power UV photoreduction, hydrazine assisted reduction, electroreduction or via hydrothermal reactions [1-8]. Mostly, the reduced form of the POMs served as effective reductants of GO and/or of the metallic salt but also as capping and stabilising agent of the resulting rGO and/or metal nano-objects [1-5]. The high electrochemical and photo-electro-catalytic performances of these nanohybrids were attributed to the synergistic effect of the rGO used as excellent conducting support and the recognised electrochemical and photo-electro-catalytic properties of the POMs and the metal nano-objects. A large number of these hybrids are based on molybdenum-containing POMs because they have excellent redox properties and great potential as electrocatalysts for challenging applications such as sensors and energy storage materials $[2-4,6,7$, 26-29]. Most of the purely molybdic POM-based hybrids are obtained with the classical Keggin-type POM $\left[\mathrm{PMo}_{12} \mathrm{O}_{40}\right] 3-\left(\mathrm{PMo}_{12}\right)$ associated or not with cationic polymers. Recently, we introduced a one-step and green method for the assembly of rGO with POM-based metal organic framework (POMOF/rGO), the POMOFs used being based on $\left\{\varepsilon-\mathrm{PMo}_{8} \mathrm{MoVI}_{4} \mathrm{O}_{40-\mathrm{x}}(\mathrm{OH})_{\mathrm{x}} \mathrm{Zn}_{4}\right\}(0 \leq \mathrm{x} \leq 4)$ Keggin-type 
polyoxomolybdate building units. Indeed, the synthesis of these POMOF/rGO hybrids was carried out in pure water, at room temperature and without any additive [30]. POMOFs serve as efficient reductants for GO as well as stabilising agents for the resulting POMOF/rGO hybrids which revealed to present high quality rGO, remarkable electrochemical properties and very high stability in aqueous media. However, besides this effective green method to assemble $\mathrm{rGO}$ and POMOF, the development of milder synthetic protocols of such polyoxomolybdate nanohybrids remains a challenge.

Herein, we introduce highly effective energy saving synthetic routes for a new generation of polyoxomolybdate nanohybrids based on the simple polyanion $\left[\mathrm{Mo}_{4} \mathrm{O}_{8}(\mathrm{OH})_{2}\left(\mathrm{H}_{2} \mathrm{O}\right)_{2}\left(\mathrm{C}_{4} \mathrm{O}_{4}\right)_{2}\right]^{2-}(\mathrm{MoS})$

and rGO, with an option of associating with Au NPs. As for POMOFs, MoS can act as a reductant but its synthesis is based on a one-pot procedure in water at room temperature, easier and quicker than the synthesis of POMOFs which is performed in an autoclave under hydrothermal conditions for several days with a purification step [30]. The reactants are relatively cheap, and the synthesis can be performed on a gram scale MoS thus possesses the properties required for low cost and effective largescale preparation and stabilization of nanohybrids. The synthesis in pure water via green one-step synthetic routes as well as the characterizations of the MoS/rGO and MoS/Au NPs composites are reported. In addition, PDDA/MoS/PDDA/GO $(\mathrm{PDDA}=$ poly $($ diallyldimethylammonium $), \mathrm{PDDA} / \mathrm{MoS}$ and PDDA/GO layer by layer films were synthesized and studied in order to evidence potential synergistic effects between the components on the electrocatalytic properties of such hybrids.

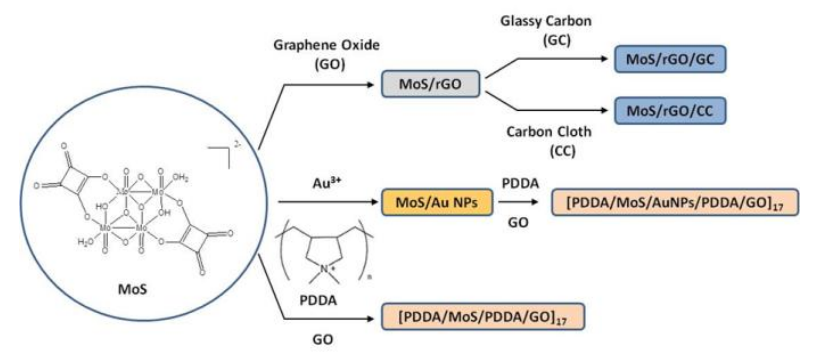

Scheme 1. Synthetic pathways for the various nanohybrids.

DFT calculations of the interaction between MoS and graphene were also performed in order to apprehend the interaction between these components. The electrocatalytic performances for the reduction of hydrogen peroxide of the MoS/rGO nanohybrids as well as preliminary tests as supercapacitors are finally reported.

\section{Experimental Section}

\section{Materials}

Hydrochloric acid, ethanol, ether, sulfuric acid, lithium chloride, potassium permanganate and sodium nitrate were purchased from Sinopharm Chemical Re- 
agent Co., Ltd.. Chloroauric acid, sodium borohydride, sodium molybdate dehydrate and ascorbic acid were obtained from Aladdin. 5\% Nafion (purchased from Sigma Aldrich) were dissolved in isopropyl alcohol to get the $0.5 \%$ Nafion to use. The other reagents were of analytical purity and were used without further purification.

\section{Synthesis}

The synthetic pathways and the abbreviations of the various materials are shown in Scheme 1.

\section{$\mathrm{Li}_{0.5} \mathrm{Na}_{1.5}\left[\mathrm{MoV}_{4} \mathrm{O}_{8}(\mathrm{OH})_{2}\left(\mathrm{H}_{2} \mathrm{O}\right)_{2}\left(\mathrm{C}_{4} \mathrm{O}_{4}\right)_{2}\right] \cdot 9 \mathrm{H}_{2} \mathrm{O}$}

(LiNa-MoS). LiNa-MoS was synthesized following a slight modification of the procedure reported previously by some of us [31]. $\mathrm{Na}_{2} \mathrm{MoO}_{4} \cdot 2 \mathrm{H}_{2} \mathrm{O}(1.0 \mathrm{~g}, 4.15$ $10^{-3} \mathrm{~mol}$ ) was dissolved in $10 \mathrm{~mL}$ of water. $\mathrm{NaBH}_{4}$ $\left(0.080 \mathrm{~g}, 2.11 \times 10^{-3} \mathrm{~mol}\right)$ was then added and the $\mathrm{pH}$ was adjusted to 7.0 with $1 \mathrm{M} \mathrm{HCl}$. The resulting brownish solution with a dark precipitate was stirred for 5 min. A solution of $\mathrm{H}_{2} \mathrm{C}_{4} \mathrm{O}_{4}\left(0.228 \mathrm{~g}, 2.0 \times 10^{-3} \mathrm{~mol}\right)$ in $10 \mathrm{~mL}$ of water was added which lead to the dissolution of the precipitate. The $\mathrm{pH}$ was adjusted to 2 with $1 \mathrm{M} \mathrm{HCl}$ and the solution stirred for another $15 \mathrm{~min}$. $\mathrm{LiCl}\left(1.0 \mathrm{~g}, 23.6 \times 10^{-3} \mathrm{~mol}\right)$ was then added and the solution was left at room temperature for $24 \mathrm{~h}$. The orange solid was filtrated on a glass filter and washed with $\mathrm{EtOH}$ and $\mathrm{Et}_{2} \mathrm{O}(\mathrm{m}=0.270$ g, yield $26 \%$ based on
Mo). IR: $v\left(\mathrm{~cm}^{-1}\right)=1805(\mathrm{~m}), 1643$ (s), 1502 (s), 1437 (s), 1091 (w), 1064 (w), 962 (m), 939 (m), 709 (m), 613 (w), 476 (w). Anal. Calc. for $\mathrm{C}_{8} \mathrm{H}_{24} \mathrm{Li}_{0.5} \mathrm{Mo}_{4} \mathrm{Na}_{1.5} \mathrm{O}_{29}$ (F.W. $1006.0 \mathrm{~g} \mathrm{~mol}^{-1}$ ) (found): $\mathrm{C}$ 9.55 (8.67), H 2.40 (1.73), Li 0.35 (0.55), Mo 38.18 (38.97), Na 3.43 (3.51). The study of the stability of MoS in water was performed by dissolving LiNa-MoS (31 mg) in $10 \mathrm{~mL}$ of water. The solution was stirred in air at room temperature for 2 to 4 days. The solution was evaporated to dryness and the IR spectrum of the powder recorded.

Synthesis of GO. GO was formed by oxidation of natural graphite flakes by a modified Hummers method [32] using $\mathrm{H}_{2} \mathrm{SO}_{4}, \mathrm{NaNO}_{3}$ and $\mathrm{KMnO}_{4}$ in an ice bath [33]. The resulting homogeneous brown GO dispersion was tested to be stable for several months and used for reduction.

Synthesis of MoS/rGO. In a typical experiment, MoS (26 mg) was dissolved in $20 \mathrm{~mL}$ of water and stirred until a yellow clear solution was obtained. Then, this solution was mixed to an aqueous suspension of GO (20 mL, $0.25 \mathrm{mg} \mathrm{mL}^{-1}$ ), and kept at room temperature for at least 4 hours, affording a dark blue suspension. The suspension was filtrated, washed thoroughly with pure water and dried.

Synthesis of MoS /Au NPs. A HAuCl 4 aqueous solu- 
tion (45 ? ?L, $0.02 \mathrm{M}$ ) (Au acid) was added to an aqueous solution of $\operatorname{MoS}(6 \mathrm{~mL}, 1.5 \mathrm{mM})(\gamma=\mathrm{n}(\mathrm{MoS}) / \mathrm{n}(\mathrm{Au}$ acid $)=10$ ). The color changed from yellow to dark red, indicating the formation of MoS stabilized Au NPs (MoS/Au NPs) which was further characterized by UV-Vis spectra. The MoS/Au NPs were separated from the reaction mixture by centrifugation and washed three times with deionized water in order to remove the residual MoS. The collected MoS/Au NPs were re-dispersed in water using mild ultrasonication to generate a stable solution of MoS/Au NPs for analysis and further utilization.

Preparation of the modified electrodes. The electrode material was either a polished glassy carbon disk (GC) or a carbon cloth (CC) plate. Prior to the modification process the CC plate was pre-treated according to the literature method to remove impurities on its surface [34]. CC was refluxed in concentrated $\mathrm{HNO}_{3}$ at $100{ }^{\circ} \mathrm{C}$ for 2 hours and then thoroughly washed with water and ethanol. Typically, MoS/rGO was dispersed in ethanol, and then grinded and sonicated to form a uniform catalyst ink (1.67 mg $\left.\mathrm{mL}^{-1}\right)$. The modified electrode was prepared by depositing $30 \mathrm{~L}$ per $\mathrm{cm}^{2}$ onto GC or CC (i.e., the MoS/rGO loading was $0.050 \mathrm{mg} \mathrm{cm}^{-2}$ ).

Fabrication of the Multilayer Films Containing MoS, MoS/Au NPs and GO. The substrates (ITO-coated glass slides or quartz) were put in an aqueous solution containing PDDA

(PDDA $=$ poly(diallyldimethylammonium), $8.0 \mathrm{wt} \%$ ) for $20 \mathrm{~min}$ to obtain a positively charged surface of PDDA layer, which can adsorb the negatively charged species (abbreviated NCS) MoS, GO and/or MoS/Au NPs via electrostatic interactions. The substrates with PDDA layer were transferred to solutions containing the NCS for $20 \mathrm{~min}$. After rinsing thoroughly with deionized water and drying with nitrogen the decorated substrates with a NCS layer were shifted to the PDDA solution (8.0 wt \%) again, leading to the adsorption of another PDDA layer. When the obtained modified substrates were put in NCS and PDDA solutions alternately, the multilayer films containing NCS and PDDA were formed. The thickness of the multilayer films could be easily adjusted by the number of deposited layers.

\section{Physical Characterization Techniques.}

FT-IR spectra were performed on a Bruker Vertex 80v FT-IR spectrometer equipped with a DTGS detector (32 scans) with a resolution of $4 \mathrm{~cm}^{-1}$ on $\mathrm{KBr}$ pellets. X-ray photoelectron spectroscopy (XPS) spectra were measured on an ESCALAB-250 spectrometer with a monochromic X-ray source (Al K $\alpha$ line, 1486.6 eV). Powder X-ray diffraction (XRD) data were acquired on a Rigaku X-ray diffractometer using $\mathrm{Cu} \mathrm{K} \alpha$ radiation at a wavelength of $1.542 \AA$. Transmission 
electron microscopy (TEM) images were recorded on a Hitachi H8100 electron microscope. Dynamic light scattering (DLS) experiments were performed on a DAWN Enhanced Optical System (DAWN EOS, Wyatt Technology Corporation). UV-vis absorption spectra were recorded on a Shimadzu UV-3100 spectrometer, and the slit width was set at $2 \mathrm{~nm}$. Zeta potential measurements were recorded on a Nano-ZS instrument, model ZEN 3600 (Malvern Instruments).

Electrochemical Characterizations. The electrochemical setup was a CHI workstation driven by a PC with the CHI660E software. All experiments were performed at room temperature. The solutions were thoroughly deoxygenated for at least $30 \mathrm{~min}$ with pure argon and kept under a positive pressure of this gas during the experiments. The working electrode material was either well-cleaned GC or CC. Potentials are quoted against a saturated calomel electrode (SCE), in a compartment separated from the test solution by a fine porosity glass frit. The counter electrode was a platinum plate with large surface area in a compartment separated from the test solution by a medium-porosity glass frit. The composition and $\mathrm{pH}$ of the media used for the electrochemical or electrocatalytical experiments were as follows: $0.5 \mathrm{M}$ $\mathrm{H}_{2} \mathrm{SO}_{4}$ solution or $0.5 \mathrm{M} \mathrm{Na}_{2} \mathrm{SO}_{4} / \mathrm{H}_{2} \mathrm{SO}_{4}$ (pH 2). Static and dynamic light scattering (SLS and DLS) measure- ments were performed by a Brookhaven Instruments Inc. light scattering spectrometer at 90 degree scattering angle at room temperature. Zeta potential analysis was performed by a Brookhaven Instruments Inc. ZetaPALS analyser. Specific experimental details for the physical characterizations are provided in the Supporting Information.

\section{RESULTS AND DISCUSSION}

Synthesis, physical and electrochemical characterizations

\section{Synthesis and Characterization of LiNa-MoS.}

The synthesis of $\mathrm{Li}_{0.5} \mathrm{Na}_{1.5}\left[\mathrm{MoV}_{4} \mathrm{O}_{8}(\mathrm{OH})_{2}\left(\mathrm{H}_{2} \mathrm{O}\right)_{2}\left(\mathrm{C}_{4} \mathrm{O}_{4}\right)_{2}\right] \cdot 9 \mathrm{H}_{2} \mathrm{O}$ (LiNa-MoS) was performed by the reaction of sodium molybdate, sodium borohydride and squaric acid. $\mathrm{NaBH}_{4}$ is a green reducing agent compared to the highly toxic hydrazine which was used in our previous synthetic protocol [31]. A panel of techniques (see experimental section) evidenced that $\mathrm{MoS}$ is the analogue of $\mathrm{LiNa}\left[\mathrm{MoV}_{4} \mathrm{O}_{8}(\mathrm{OH})_{2}\left(\mathrm{H}_{2} \mathrm{O}\right)_{2}\left(\mathrm{C}_{4} \mathrm{O}_{4}\right)_{2}\right] \cdot 2 \mathrm{H}_{2} \mathrm{O}$ and possesses a tetranuclear $\left[\mathrm{Mo}_{4} \mathrm{O}_{8}(\mathrm{OH})_{2}\left(\mathrm{H}_{2} \mathrm{O}\right)_{2}\left(\mathrm{C}_{4} \mathrm{O}_{4}\right)_{2}\right]^{2-}$ core, (Figure 1). The tetranuclear anion is built up of four edge sharing $\mathrm{Mo}^{\mathrm{v}}$ octahedra, separated in two pairs corresponding to $\mathrm{Mo}^{\mathrm{V}}-\mathrm{Mo}^{\mathrm{V}}$ dimers with localized metal-metal bonds. Two squarate ligands bridge two Mo 
ions of two different dimers. Two $\left[\mu_{3}-\mathrm{O}\right.$ atoms are located at the junction of three Mo octahedra leading to a compact and robust structure.

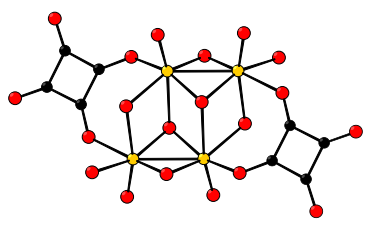

a)

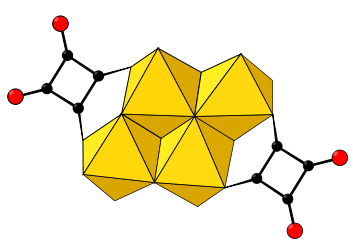

b)
Figure 1. a) Ball-and-stick and b) polyhedral representations of the structure of the MoS anion; yellow sphere $=$ Mo, red spheres $=0$, black spheres $=\mathrm{C}$.

The long-term stability of MoS in pure water as a function of time was studied by monitoring the evolution of its IR spectrum over several days (Figure S1). When MoS was dissolved in water and the solution stirred in air, the orange solution progressively turned blue. A blue precipitate was obtained by evaporation after 2 or 4 days respectively and its IR spectrum recorded. The blue color indicates the partial oxidation of $\mathrm{Mo}^{\mathrm{V}}$ ions and the formation of mixed-valenced species. However, while broadened, the position of the peaks in the IR spectra does not change compared to those of the MoS precursor, showing that the structure is globally maintained. It is noted that we didn't observe the formation of POM-based colloids. Indeed, SLS and DLS measurements indicate that the MoS nanoclusters exist as single clusters in solution, as the scattered inten- sity from MoS solutions (determined by the SLS) is very low (no supramolecular structure formation) and the DLS measurements do not detect any large structures in solution. From the Zeta potential analysis studies, in $1.0 \mathrm{mg} \mathrm{mL}^{-1}$ aqueous solution each $\mathrm{MoS}$ cluster carries -1.55 effective charges as the additional charges have been neutralized by closely-associated counterions.

\section{Synthesis and Characterization of MoS/rGO. Specific} experimental details for $\mathrm{GO}$ and $\mathrm{MoS} / \mathrm{rGO}$ are provided in the Experimental section. The synthesis of MoS/rGO hybrids was carried out by reaction of the desired amount of MoS with an aqueous exfoliated dispersion of GO at room temperature without the requirement of any additive. During the reduction process, the light brown-colored suspension turned blue-black after about 4 hours. These observations underscore the high efficiency of the reduction reaction yielding the well-known black color of rGO nanosheets and the blue-colored $\mathrm{Mo}^{\mathrm{V}}-\mathrm{Mo}^{\mathrm{VI}}$ mixed-valenced species resulting from partial oxidation of MoS. These observations suggest that MoS/rGO nanohybrids were obtained, in line with the reported ones for various POM/rGO nanohybrids [2-7]. Figure S2a shows the UV/VIS-NIR spectra of GO, MoS and the as-prepared $\mathrm{MoS} / \mathrm{rGO}$ in pure water. The comparison of these spectra evidences that after complete reduc- 
tion, the spectrum of the sample exhibits the spectral fingerprint of the mixed-valenced species featured by a peak located at $775 \mathrm{~nm}$ followed by a shoulder at ca. $670 \mathrm{~nm}$ (Figure S2b). Moreover, the absorption of rGO should contribute to the observed absorption increase at higher wavelengths.

To obtain pure MoS/rGO, the suspension was filtrated, washed with pure water, and dried. The black precipitate was characterized by various physical techniques and cyclic voltammetry (CV). Figure 2a exhibits the typical FESEM image of the MoS/rGO nanohybrid. As expected the image features rGO nanosheets looking like crumpled silk veil waves, which confirms the successful reduction of GO. The TEM (Figures $2 \mathrm{~b}, 2 \mathrm{c}$ with different magnifications) and HRTEM (Figure 2d) images show that a majority of nanoclusters measuring $5 \pm 1 \mathrm{~nm}$ in diameter are uniformly dispersed on transparent rGO nanosheet. The energy-dispersive X-ray spectrum (EDS) elemental mapping images (Figures 2e, 2f, 2g) on carbon, oxygen, and molybdenum confirm their homogeneous distribution in MoS/rGO. The oxygen mainly comes from the residual oxygen containing groups in $\mathrm{rGO}$ and the oxygen of the POM.

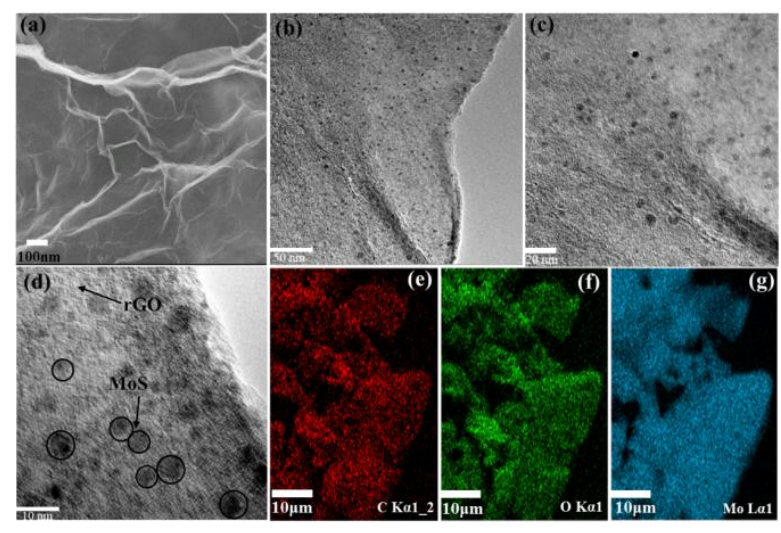

Figure 2. (a) Field-emission scanning electron microscopy (FESEM), (b, c) different magnifications of TEM and (d) HRTEM images of MoS/rGO; (e-g) energy-dispersive X-ray spectrum (EDS) elemental mapping images of MoS/rGO.

The degree of GO reduction and the chemical composition of MoS/rGO were monitored by XPS. Figures $3 a$ and $3 b$ feature the XPS C 1s spectra of GO and $\mathrm{MoS} / \mathrm{rGO}$ respectively. Table S1 gathers the contents of the different types of carbon obtained with GO and MoS/rGO. The C 1s deconvolution spectrum of GO features the well-known four types of carbon which appear at $284.8 \mathrm{eV}$ (graphite-like $\mathrm{C}, \mathrm{C}-\mathrm{C} / \mathrm{C}=\mathrm{C}$ ), $286.8 \mathrm{eV}$ $(\mathrm{C}-0), 287.8 \mathrm{eV}(\mathrm{C}=0)$ and $289.0 \mathrm{eV}(\mathrm{O}-\mathrm{C}=0)$ respectively. The peak intensities of the $\mathrm{C}-\mathrm{O}$ and $\mathrm{C}=\mathrm{O}$ oxygen containing groups decreased after reduction of GO by MoS, indicating that most of these groups are removed. The increase of the graphitic-like C content (from 39.1\% to $73.1 \%$ ) is attributed to the expected restoration of $\mathrm{sp}^{2}$ carbon network after the reduction process. The XPS analysis also indicates the presence of molyb- 
denum in the valence states V and VI with the VI state representing the largest amount (Figure 3c). Altogether, these observations confirm that MoS serves for formation of MoS/rGO nanohybrids during the reduction of GO by MoS.
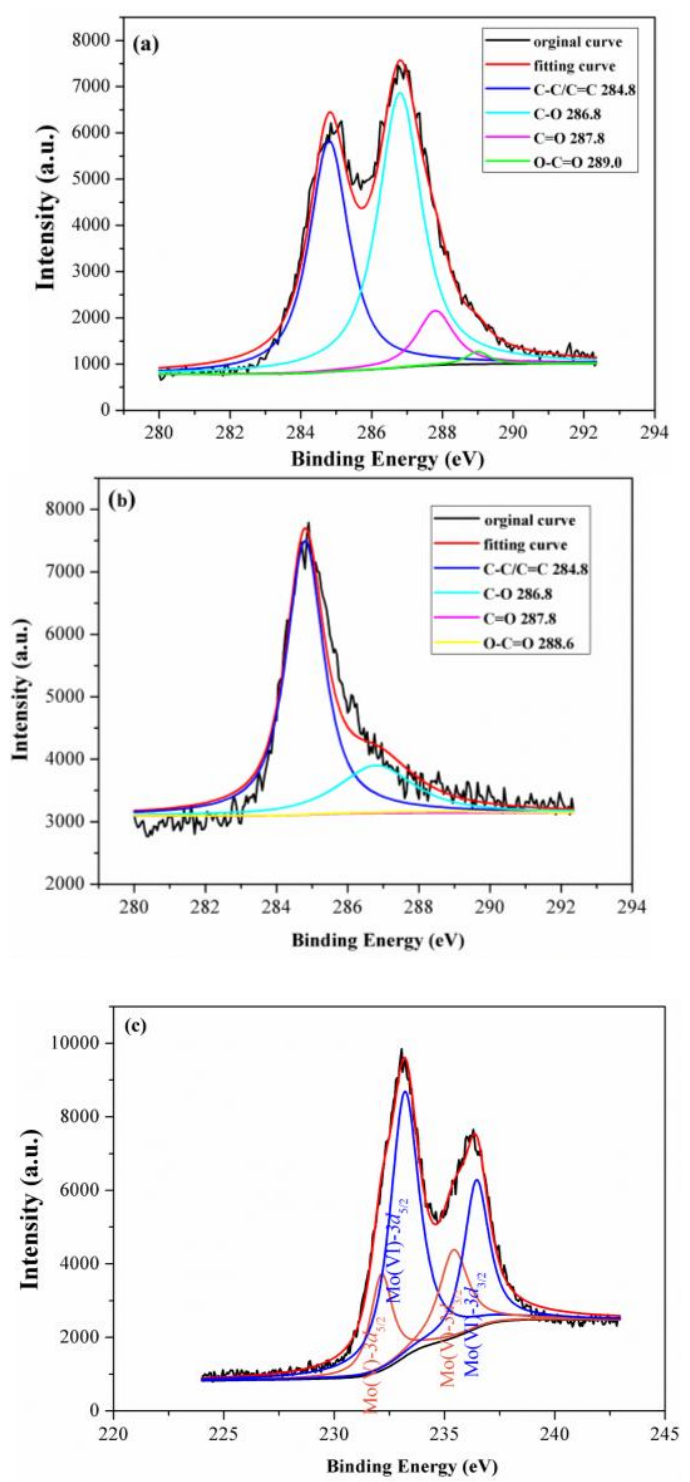

Figure 3. C 1s XPS spectra of (a) GO and (b) $\mathrm{MoS} / \mathrm{rGO}$; (c) XPS spectra of Mo 3d in MoS/rGO.

Raman Spectroscopy is one of the most appropriate techniques to probe the defects and structural proper- ties of carbon materials [35]. Figure 4 shows the $\mathrm{G}$ and D bands of the Raman spectra of MoS/rGO, GO and natural G. The G band is attributed to the first-order scattering of the E2g vibration mode observed for the sp2 domains while the D band is a breathing mode of k-point phonons of A1g symmetry [35]. In particular, the intensity ratio of $\mathrm{D}$ and $\mathrm{G}$ bands $\left(I_{D} / I_{G}\right)$ provides disorder degree and average size of the $\mathrm{sp}^{2}$ domains. As expected, the $I_{D} / I_{G}$ intensity ratio of GO (0.949) is much higher than that of $G(0.129)$ indicating the presence of defects and the diminution in size of the $\mathrm{sp}^{2}$ domains due to the extensive oxidation process. For $\mathrm{MoS} / \mathrm{rGO}$, the $I_{D} / I_{G}$ intensity ratio increases to 0.970 indicating a slight decrease in the average size of the small $\mathrm{sp}^{2}$ domains upon reduction of GO [36]. This observation and is usually attributed to the formation of a larger number of the small $\mathrm{sp}^{2}$ domains [36]

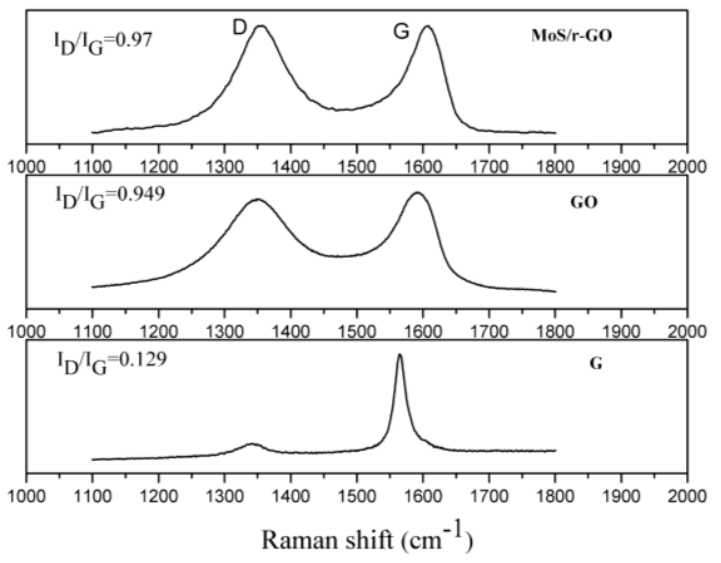

Figure 4. Raman spectra of natural G, GO and $\mathrm{MoS} / \mathrm{rGO}$. 
X-ray diffraction (XRD) measurements have been performed on GO and MoS/rGO (Figure S3). As expected, the XRD powder pattern of GO exhibits an intense peak located at $2 \theta=11.7^{\circ}$ correlated to a layered structure with an interlayer distance of ca. $7.56 \AA$. The absence of this peak in MoS/rGO confirms the reduction of GO by the POM. The broad peak at ca. $2 \theta=26^{\circ}$ corresponds to an interlamellar d-spacing of $3.42 \AA$.

The MoS/rGO nanohybrid immobilized on glassy carbon $(\mathrm{MoS} / \mathrm{rGO} / \mathrm{GC})$ were further characterized by solid state cyclic voltammetry (CV) in $0.5 \mathrm{M} \mathrm{H}_{2} \mathrm{SO}_{4}$ medium. Figure 5 displays three well-defined chemically reversible reduction waves, symbolized as I to III, associated to the $\mathrm{Mo}^{\mathrm{VI}}$ centers within MoS/rGO. The mean peak potentials $E_{1 / 2}=\left(E_{p a}+E_{p c}\right) / 2$ for $\mathrm{MoS} / \mathrm{rGO} / \mathrm{GC}$ appeared at about $+0.306 \mathrm{~V}\left(\mathrm{I}-\mathrm{I}^{\prime}\right)$, $+0.198 \mathrm{~V}\left(\mathrm{II}-\mathrm{II}^{\prime}\right)$ and $-0.022 \mathrm{~V}\left(\mathrm{III}-\mathrm{III}^{\prime}\right)$ vs. SCE with peak potential separations of 10,14 and $21 \mathrm{mV}$ (at scan rate: $100 \mathrm{mV} \cdot \mathrm{s}^{-1}$ ), respectively. These data are consistent with those reported in the literature and are attributed to $\mathrm{Mo}^{\mathrm{VI}} \rightarrow \mathrm{Mo}^{\mathrm{V}}$ redox processes [37-40]. Figure 5 also shows the variation of the CV characteristics as a function of the potential scan rate. These CVs exhibit symmetrical shape featuring the reversible reduction and oxidation of surface confined reactants. The dependence of cathodic peak current intensity of the second wave as a function of the scan rate is also shown in the inset in Figure 5. The linearity $(R=0.999)$ of these curves confirms the surface-confined character of the observed waves. Altogether, the electrochemical observations also underline the successful synthesis of remarkably stable MoS/rGO nanohybrids. In contrast, ill-defined or moderately stable redox waves were reported for $\mathrm{PMo}_{12} / \mathrm{rGO}$ prepared via mere adsorption in water at room temperature without any toxic additive or sonification process [27].

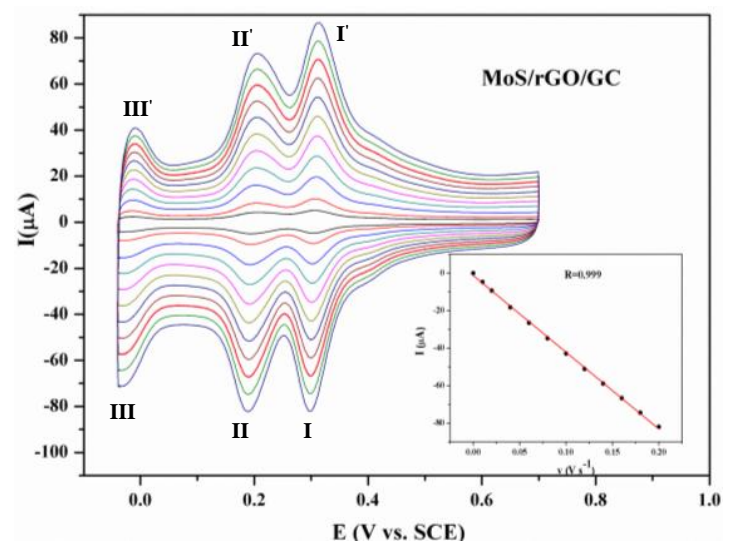

Figure 5. Cyclic voltammograms (CVs) and peak current intensity variations for $\mathrm{MoS} / \mathrm{rGO} / \mathrm{GC}$ in $0.5 \mathrm{M}$ $\mathrm{H}_{2} \mathrm{SO}_{4}$ medium. The reference electrode was a saturated calomel electrode (SCE). CVs as a function of scan rate (from inner to outer: 10, 20, 40, 60, 80, 100, $120,140,16,180$ and $200 \mathrm{mV} \mathrm{s}^{-1}$ ); (inset) the dependence of cathodic current of the second reduction wave as a function of the scan rate.

\section{DFT calculations of the interaction of $\mathrm{MoS}$ or $\mathrm{PMo}_{12}$}

\section{and graphene}

It is well-known that most POMs have the propensity to spontaneously self-assemble onto different carbon materials $[2,3,41,42]$. This adsorption is at- 
tributed to an electrostatic interaction which proceeds through proton transfer from the polyoxometalates to the oxygen-containing group present on these materials. Recent DFT studies reported by some of us demonstrate that the strength of the interaction between the POM and the carbon material depends strongly on the nature of the POM [5]. Therefore, the easier synthesis of $\mathrm{MoS} / \mathrm{rGO}$ compared to that of $\mathrm{PMo}_{12} / \mathrm{rGO}$ could be due, at least partly, to the extent of the interaction. The interaction between MoS or $\mathrm{PMo}_{12}$ and graphene were investigated based on DFT calculations, which were performed by using the SIESTA (Spanish Initiative for the Electronic Simulations of Thousand of Atoms) program package [43-45] The geometry and energy properties were described using general gradient approximation (GGA) in the scheme of Perdew-Burke-Ernzerhof (PBE) [46], and the double- $\xi$ basis set plus polarization (DZP) function was used for all elements. The cut off energy of the plane-wave basis is $200 \mathrm{eV}$, and $3 \times 3 \times 1 \mathrm{k}$-point meshes were used for the Brillouin zone integration. The atomic relaxation is carried out until forces are less than $0.1 \mathrm{eV} / \AA$, while the energy properties are carried out until forces are less than $0.02 \mathrm{eV} / \AA ̊$. The geometries were investigated by standard density functional theory (DFT) using the general gradient approximation (GGA) of Perdew, Burke, and Ernzerhof (PBE).
The adsorption energy for the most stable configuration (Figure 6) is $-1.97 \mathrm{eV}$, indicating that the adsorption process of $\left[\mathrm{MoV}_{4} \mathrm{O}_{8}(\mathrm{OH})_{2}\left(\mathrm{H}_{2} \mathrm{O}\right)_{2}\left(\mathrm{C}_{2} \mathrm{O}_{4}\right)_{2}\right]^{2-}$ on graphene is very strong. The same results can also be drawn from the distance (3.04 $\AA$ ) and the charge transfer (0.86 e). The adsorption energy and the charge transfer for all cases in our calculations is much larger than that of $\left[\mathrm{PMo}_{12} \mathrm{O}_{40}\right]^{3-}(-1.50 \mathrm{eV}$ and $0.08 \mathrm{e})$, indicating that the adsorption process of $\left[\mathrm{MoV}_{4} \mathrm{O}_{8}(\mathrm{OH})_{2}\left(\mathrm{H}_{2} \mathrm{O}\right)_{2}\left(\mathrm{C}_{2} \mathrm{O}_{4}\right)_{2}\right]^{2-}$ on graphene is easier than for $\left[\mathrm{PMo}_{12} \mathrm{O}_{40}\right]^{3-}$. Additionally, we also considered the neutral structure of the tetranuclear POM, adding two $\mathrm{Na}^{+}$to compensate the negative charge. The adsorption energy and charge transfer between the neutral POM and graphene are $-1.06 \mathrm{eV}$ and $0.24 \mathrm{e}$, respectively. Therefore, the DFT calculation results are consistent with the experimental observations and suggest that the MoS/rGO hybrids should be good candidates as highly stable and effective electrode materials.

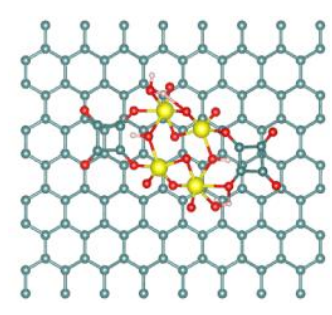

Top view

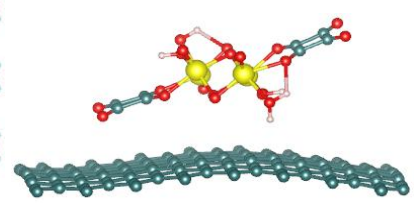

Side view
Figure 6. Ball-and-Stick representation of the most stable optimized structure for MoS/rGO. Colour code: Mo (yellow), O (red), C (blue), H (pink). 
Preparation and Characterization of MoS/Au NPs and of multilayers films

Specific experimental details for $\mathrm{GO}$ and $\mathrm{MoS} / \mathrm{Au}$ NPs are provided in the Experimental Section. After the $\mathrm{Au}$ acid was mixed with MoS, the electron exchange process from MoS to Au acid occurred spontaneously at room temperature without any additives, with a color change of the solution from yellow to dark red within $30 \mathrm{~s}$. It follows that $\mathrm{Mo}^{\mathrm{V}}$ centers in $\mathrm{MoS}$ were oxidized to $\mathrm{Mo}^{\mathrm{VI}}$ while $\mathrm{Au}$ acid was reduced from $\mathrm{Au}^{\mathrm{III}}$ to $\mathrm{Au}^{0}$, resulting in the formation of $\mathrm{Au}$ NPs. The solution UV-vis spectra of $\mathrm{Au}$ acid (curve a), MoS (curve b) and MoS/Au NPs (curve c) are displayed in Figure S4. It can be noted in curve $\mathrm{c}$ that a new absorption band at ca. $550 \mathrm{~nm}$, characteristic of the Au NPs, is observed after mixing $\mathrm{Au}$ acid and MoS. The presence of a single-band suggests that the NPs are spherical. Moreover, the colloid solution of MoS/Au NPs is very stable and does not present any precipitate after two months without adding any stabilizer, which indicates that MoS serves both as high-performance reductant and stabilizer.

Figures 7a-c show representative TEM images at different magnifications of MoS/Au NPs deposited on a carbon-coated copper grid. Most of these nanoparticles are spherically shaped and quasi-monodisperse with an average diameter of ca. $36 \mathrm{~nm}$ calculated by a statistical study of $60 \mathrm{NPs}$, which is consistent with the results of DLS analysis (the hydrodynamic radius was ca. $37 \mathrm{~nm}$ ). Figure 7c exhibits a magnified image of a MoS/Au NP nanoparticle which highlights a thin layer of MoS (ca. $2 \mathrm{~nm}$ thickness), suggesting the core-shell architecture of MoS/Au NPs. Moreover, the HRTEM image featured in Figure7d displays the lattice fringe of $\mathrm{Au}$ crystalline face $(0.23 \mathrm{~nm})$, indicating a highly monocrystalline feature of MoS/Au NPs. In addition, in situ EDX analysis detects the molybdenum and gold signals (Figure S5), further demonstrating the presence of a MoS layer around Au NPs.

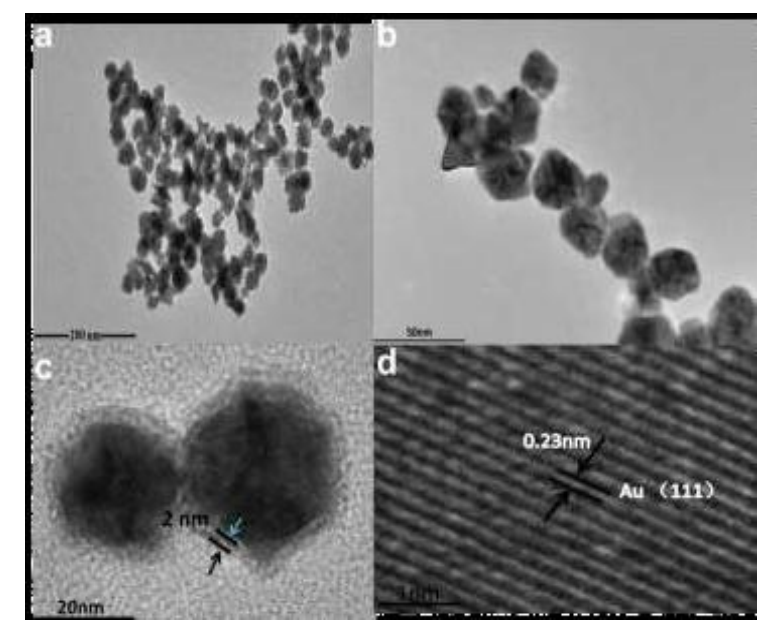

Figure 7. (a-c) different magnifications of TEM images of MoS/Au NPs; (d) HRTEM image of MoS/Au NPs.

XPS was used to further confirm the nature of the prepared MoS/Au NPs. For this purpose, a cleaned sample was dropped on silicon substrate. Figure S6 shows Au $4 \mathrm{f}$ and Mo 3d spectra of MoS/Au NPs. On one 
hand, the characteristic $\mathrm{Au} 4 \mathrm{f}$ peaks for Au0, ascribed to $4 f_{7 / 2}$ and $4 f_{5 / 2}$ levels respectively, appear at 83.7 and $87.5 \mathrm{eV}$. The characteristic $\mathrm{Au} 4 \mathrm{f}$ peaks for $\mathrm{Au}^{\mathrm{III}}$ are not observed, demonstrating the effectiveness of the reduction process from $\mathrm{Au}^{\mathrm{III}}$ to $\mathrm{Au}^{0}$ performed by $\mathrm{MoS}$. On the other hand, after the sample was washed completely, two characteristic Mo 3d peaks at ca. 232.3 and $235.6 \mathrm{eV}$ are also detected, further supporting our proposal that MoS acts both as reducing and capping molecules for the synthesis of Au NPs.

As an alternative method, we have also shown that the well-known layer by layer (LBL) assembly technique can be employed to prepare multilayer assemblies of MoS, GO and/or Au NPs by using PDDA as a cationic moiety linker. The multilayer film growth process on quartz substrates was checked by UV-vis absorption spectra. For comparison purpose, two multilayer films containing GO, MoS and MoS/Au NPs were fabricated using identical experimental conditions. Figure S7 shows the changes of UV-vis absorption spectra for two multilayer films on quartz slides. Comparing Figures S4, S7and S8, it is clear that the characteristic bands of MoS, GO and MoS/Au NPs are globally maintained in the multilayer films, indicating that their electronic structures are retained. The small shifts observed are attributed to the electrostatic interactions between GO, MoS, MoS/Au NPs and PDDA together with the different chemical environments around these species. In addition, as shown in the insets of Figure S7, the absorbance values for the characteristic bands of MoS and GO increase with the increase of the layer number. Linear dependences are observed in the absorbances at $235 \mathrm{~nm}$ corresponding to GO and $310 \mathrm{~nm}$ corresponding to MoS, demonstrating the nearly uniform growth of the two multilayer films.

\section{MoS/rGO based cathode material as high-performance electrocatalyst and promising supercapacitor}

Owing to its electrochemical properties and stability, the MoS/rGO-based electrode material may be a potential candidate for triggering several effective and clean electrocatalytic processes and for energy storage. The applications of MoS/rGO as electrocatalyst for the reduction of hydrogen peroxide reduction and as cathode for supercapacitor were selected to exemplify the bifunctional properties of MoS/rGO.

\section{Electrocatalysis of hydrogen peroxide reduction}

The effective electrocatalytic detection of $\mathrm{H}_{2} \mathrm{O}_{2}$ is crucial in medical, environmental and industrial fields. In particular, $\mathrm{H}_{2} \mathrm{O}_{2}$ is a by-product of many oxidative biological reactions, including those of glucose oxidase, cholesterol oxidase, alcohol oxidase, galactose oxidase, sarcosine oxidase and l-amino-acid oxidase. 
$\mathrm{CV}$ and amperometric measurements were used to evaluate the electrocatalytic activity of $\mathrm{MoS} / \mathrm{rGO} / \mathrm{GC}$ towards $\mathrm{H}_{2} \mathrm{O}_{2}$ reduction in a pH 2 medium. The loading amount of MoS/rGO was $0.05 \mathrm{mg} \mathrm{cm}^{-2}$. Figure 8a displays the $\mathrm{CV}$ responses of MoS/rGO/GC to additions of $\mathrm{H}_{2} \mathrm{O}_{2}$ over the $0-20 \mathrm{mM}$ range. It can be clearly seen that the reduction of $\mathrm{H}_{2} \mathrm{O}_{2}$ is efficiently triggered by the Mo-waves of MoS/rGO as evidenced by the gradual increase of the reduction currents upon increasing $\mathrm{H}_{2} \mathrm{O}_{2}$ concentration. Importantly, the calibration curve featured in Figure 8b shows that the peak current at $-0.2 \mathrm{~V}$ versus SCE increased proportionally with the $\mathrm{H}_{2} \mathrm{O}_{2}$ concentration. As shown in Figure $\mathrm{S} 9$, the [PDDA/MoS/PDDA/GO ${ }_{17}$ LBL film-modified electrode also exhibited the ability to efficiently reduce $\mathrm{H}_{2} \mathrm{O}_{2}$ which underscores the interest of the LBL assembly technique as an alternative method for MoS/rGO-based electrocatalyst preparation. Comparative studies show that the ability of the $[\mathrm{PDDA} / \mathrm{MoS} / \mathrm{PDDA} / \mathrm{GO}]_{17}$ composite to electrocatalytically reduce $\mathrm{H}_{2} \mathrm{O}_{2}$ is much higher than that of PDDA/MoS or PDDA/GO. This observation suggests that the superior performance of $[\mathrm{PDDA} / \mathrm{MoS} / \mathrm{PDDA} / \mathrm{GO}]_{17}$ is due to the synergistic effect of its components.

As a further study, the amperometric response to $\mathrm{H}_{2} \mathrm{O}_{2}$ of a MoS/rGO/GC modified electrode was inves- tigated to reinforce that this material is promising for application as a noble metal-free $\mathrm{H}_{2} \mathrm{O}_{2}$ sensor. A typical current-time plot of MoS/rGO/GC upon the successive addition of aliquots of $\mathrm{H}_{2} \mathrm{O}_{2}$ is shown in Figure 9a. It can be clearly observed that the currents increased stepwise with successive additions of $\mathrm{H}_{2} \mathrm{O}_{2}$ into the stirred electrolyte, with the current responses of about $2 \mathrm{~s}$ at $-0.2 \mathrm{~V}$ versus SCE. Importantly, the obvious increase of the current could be observed when the concentration of $\mathrm{H}_{2} \mathrm{O}_{2}$ was as low as $50 \mu \mathrm{M}$ (Figure $9 b$, a local image corresponding to the rectangular area featured in Figure 9a). The corresponding calibration curve presented in Figure 9c shows that the hydrogen peroxide sensor based on MoS/rGO exhibited a linear response range from $5 \times 10^{-5}$ to $2 \times 10^{-2} \mathrm{M}$ with a linearity regression equation (LRE) of $\mathrm{I}(\mu \mathrm{A})=4.17 \mathrm{C}$ (mM)-0.81 $\left(\mathrm{R}^{2}=0.997\right)$, a limit of detection (LOD) of 2 $\mu \mathrm{M}$ (based on $\mathrm{S} / \mathrm{N}=3$ and a sensitivity of $33.2 \mu \mathrm{A} \mathrm{cm}-2$ $\mathrm{mM}^{-1}$ ). Figure $9 \mathrm{~d}$ is a local image corresponding to the rectangular area of Figure 9c shown to highlight the linear calibration plot $\left(\mathrm{R}^{2}=1\right)$ obtained with low concentrations of $\mathrm{H}_{2} \mathrm{O}_{2}\left(5 \times 10^{-5}\right.$ to $\left.10^{-3} \mathrm{M}\right)$. This observation underscores the excellent sensitivity of MoS/rGO towards the $\mathrm{H}_{2} \mathrm{O}_{2}$ reduction reaction compared to those of POM-based reported electrocatalysts (Table S2). 

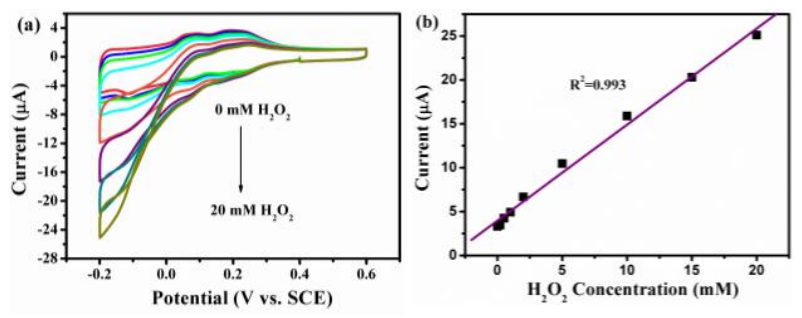

Figure 8. (a) $\mathrm{CVs}$ of the $\mathrm{MoS} / \mathrm{rGO}\left(0.05 \mathrm{mg} / \mathrm{cm}^{2}\right)$ modified GC electrode in $0.5 \mathrm{M} \mathrm{Na}_{2} \mathrm{SO}_{4}+\mathrm{H}_{2} \mathrm{SO}_{4}(\mathrm{pH}=$ 2) at $10 \mathrm{mV} \mathrm{s}^{-1}$ in the presence of $\mathrm{H}_{2} \mathrm{O}_{2}$ with various concentrations from 0 to $20 \mathrm{mM}$. (b) The corresponding calibration plot of the cathodic currents with the concentrations of $\mathrm{H}_{2} \mathrm{O}_{2}$ extractedat the potential of -0.2 $\mathrm{V}$ vs. SCE from (a).
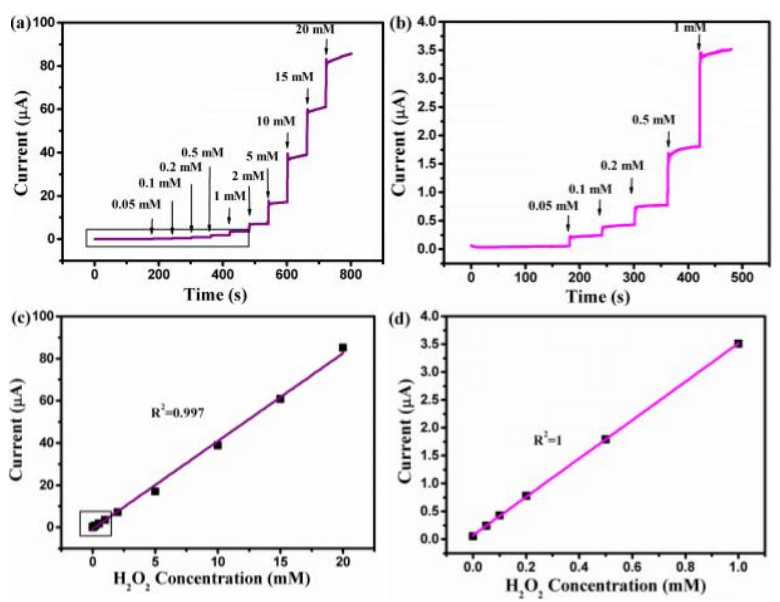

Figure 9. Typical amperometric responses of the $\mathrm{MoS} / \mathrm{rGO}$ modified GC electrode to successive addition of aliquot $\mathrm{H}_{2} \mathrm{O}_{2}$ at $-0.2 \mathrm{~V}$ vs. SCE in $0.5 \mathrm{M} \mathrm{Na}_{2} \mathrm{SO}_{4}+$ $\mathrm{H}_{2} \mathrm{SO}_{4}(\mathrm{pH}=2)(\mathrm{a})$ and the corresponding calibration plot of steady-state currents against concentrations of $\mathrm{H}_{2} \mathrm{O}_{2}$ (c). (b) and (d) are local images corresponding to the rectangular area of (a) and (c), respectively.

\section{Preliminary evaluation of MoS/rGO/CC electrode for}

\section{supercapacitor application}

Supercapacitors (SCs) are very promising energy storage devices owing to their high-power density, long cycle life time and potential application in various devices including consumer electronics and electric vehicles [47-50]. Based on different charge storage mechanisms, SCs can be categorized into electrical double-layer capacitors (EDLCs) and pseudocapacitors. There are many design approaches for SCs based on POMs and a variety of carbon materials including rGO because most POMs can undergo chemically reversible multi-electron processes in various media. However, to our knowledge, SCs made of POM/rGO nanohybrids coated on a carbon cloth substrate (POM/rGO/CC) have not been reported. For SC application, CC substrate offers significant advantages including low-cost, good electrical conductivity, low thickness, light weight, good flexibility, high mechanical stability, good chemical stability and 3D structure. Scheme 2 features the schematic preparation of $\mathrm{MoS} / \mathrm{rGO} / \mathrm{CC}$, which simply consists in the deposition of a MoS/rGO suspension, prepared as described in the experimental section, on a CC substrate.

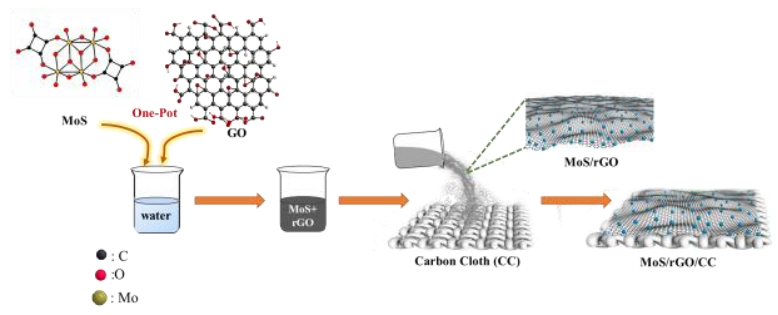

Scheme 2. Scheme of the synthesis of MoS/rGO/CC.

Figure 10 compares the CV characteristics of $\mathrm{MoS} / \mathrm{rGO} / \mathrm{CC}$ and $\mathrm{MoS} / \mathrm{rGO} / \mathrm{GC}$ recorded in $0.5 \mathrm{M}$ 
$\mathrm{H}_{2} \mathrm{SO}_{4}$ medium. The CV pattern of $\mathrm{MoS} / \mathrm{rGO} / \mathrm{CC}$ not only exhibits the general shape as that of $\mathrm{MoS} / \mathrm{rGO} / \mathrm{GC}$ but also shows that its CV area is substantially larger than that of MoS/rGO/GC and, accordingly, its capacitive response is also much higher. This can be easily explained considering that compared to the GC substrate, CC has a larger specific surface and a more porous morphology which favors ion diffusion and electron transport. As featured in Figure S10, the specific surface of bare CC is nearly 2.5 -fold smaller than that of $\mathrm{MoS} / \mathrm{rGO} / \mathrm{CC}$ which underscores the important contribution of the pseudocapacitance associated with MoS/rGO. Figure S11 displays the CV curve as a function of the scan rate for MoS/rGO/CC. The dependence of cathodic and anodic peak currents of the second wave as a function of the scan rate is also shown in the inset in the Figure S11. The linearity of these curves confirms the surface-confined characters of the observed waves which are well-defined whatever the value of the scan rate (from $10 \mathrm{mV}$ to $200 \mathrm{mV} / \mathrm{s}$ ).

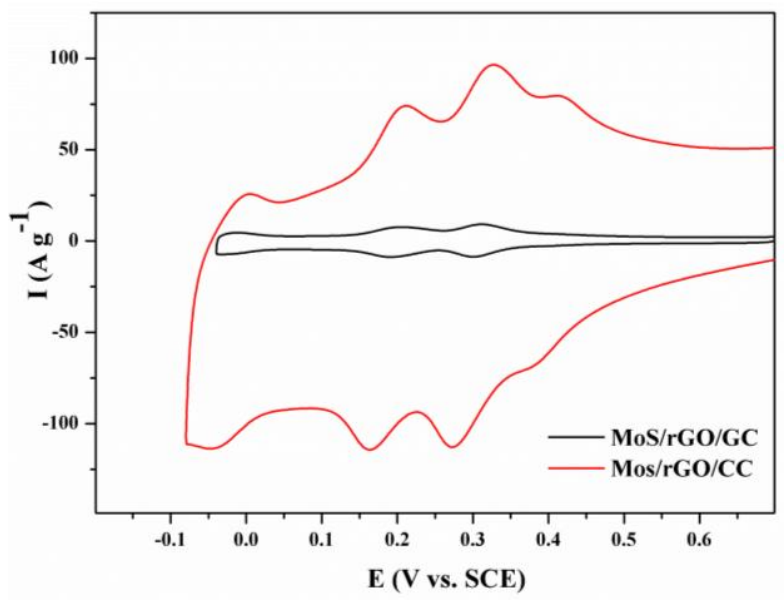

Figure 10. Superposition of the cyclic voltammograms of $\mathrm{MoS} / \mathrm{rGO} / \mathrm{CC}$ and $\mathrm{MoS} / \mathrm{rGO} / \mathrm{GC}$, run in $0.5 \mathrm{M} \mathrm{H}_{2} \mathrm{SO}_{4}$ medium at $100 \mathrm{mV} \mathrm{s}^{-1}$.

Capacitive behavior of the electrode was studied by $\mathrm{CV}$ within a potential range of +0.7 to $-0.08 \mathrm{~V}$ versus SCE at $10 \mathrm{mV} \mathrm{s}^{-1}$. The specific capacitance (Cs) was calculated by using the following equation:

$$
\mathrm{Cs}=\mathrm{Q} / \mathrm{m} \Delta \mathrm{V}
$$

where $Q$ is the cathodic voltammetric charge determined by integrating the area under the CV curve, $\mathrm{m}$ is the mass of active materials, and $\Delta \mathrm{V}$ is the width of the potential window of the CV. To evaluate the contribution of MoS/rGO to the capacitive performance of $\mathrm{MoS} / \mathrm{rGO} / \mathrm{CC}$, the $\mathrm{Cs}$ of $\mathrm{MoS} / \mathrm{rGO}$ was calculated by subtracting the charge of bare CC from that of $\mathrm{MoS} / \mathrm{rGO} / \mathrm{CC}$. The Cs due to MoS/rGO was $870 \mathrm{~F} \mathrm{~g}^{-1}$ at $10 \mathrm{mV} \mathrm{s}^{-1}$, which compares favorably with those determined from $\mathrm{CV}$ at $10 \mathrm{mV} \mathrm{s}^{-1}$ in acidic media for effective $\mathrm{PMo}_{12} /$ polymer/rGO based supercapacitor cathodes (from 406 to $650 \mathrm{~F} \mathrm{~g}^{-1}$ ) [26-28]. As stability 
is an important prerequisite for practical application, we also studied the cycling stability of MoS/rGO/CC at $100 \mathrm{mV} \mathrm{s}^{-1}$ and found that the electrode retains its initial capacitance after 5000 cycles. Therefore, the $\mathrm{MoS} / \mathrm{rGO} / \mathrm{CC}$ cathode is a promising candidate as low-cost electrode material for flexible and high-performance SC. Work is in progress to perform further evaluation of the MoS/rGO/CC electrode by using galvanostatic charging/discharging measurements.

\section{Conclusions}

In summary, nanohybrids that combine the simple and cheap $\quad\left[\mathrm{MoV}_{4} \mathrm{O}_{8}(\mathrm{OH})_{2}\left(\mathrm{H}_{2} \mathrm{O}\right)_{2}\left(\mathrm{C}_{4} \mathrm{O}_{4}\right)_{2}\right]^{2-}$ polyoxomolybdate with reduced graphene or Au nanoparticles were prepared for the first time, in water at room temperature via one-step synthetic routes. DFT calculations of the interaction between MoS and graphene demonstrate that the interaction between them is strong and even much stronger than that observed with the classical polyoxomolybdate $\mathrm{PMo}_{12}$ [43-45]. Moreover, the noble metal-free MoS/rGO hybrid displays excellent electrocatalytic performances towards hydrogen peroxide reduction with high sensitivity, large detection range and low detection limit. The specific capacitance for MoS/rGO/CC obtained from $\mathrm{CV}$ at scan rate of $10 \mathrm{mV} \mathrm{s}^{-1}$ is higher than those of previously reported POM-based supercapacitors
[27-29]. It is proposed that the association of MoS/rGO and CC induces significant enhancement of the pseudocapacitance performance of MoS/rGO. These findings open the way for facile and energy saving synthesis of novel and high- performance polyoxomolybdate-based electrode materials and their use in challenging electrocatalytic reactions or in energy storage devices.

\section{Acknowledgements}

This work was supported by the National Natural Science Foundation of China (No. 91545125, U1662121, 21173102 and 21473072), Youth Innovation Promotion Association of CAS, the CNRS, the Ministère de l'Enseignement Supérieur et de la Recherche, the Université Paris-Saclay, the Université de Versailles Saint-Quentin, the NSF (CHE1607138) and the University of Akron.

\section{References}

1 B. Keita, T. Liu, L. Nadjo, J. Mater. Chem. 19 (2009) 19.

2 S.G. Mitchell, J.M. de la Fuente, J. Mater. Chem. 22 (2012) 18091.

3 R. Liu, S. Li, G. Zhang, A. Dolbecq, P. Mialane, B. Keita, J. Clust. Sci. 25 (2014) 711.

4 U. Jameel, M. Zhu, X. Chen, Z. Tong, J. Mater Sci. 5 (2016) 2181.

5 R. Liu, G. Zhang, H. Cao, S. Zhang, Y. Xie, A. Haider, U. Kortz, B. Chen, N.S. Dalal, Y. Zhao, L. Zhi, C. Wu, L. Yan, Z. Su, B. Keita, Energy Environ. Sci. 9 (2016) 1012.

6 J. Suarez-Guevara, V. Ruiz, P. Gomez-Romero, Phys. Chem. Chem. Phys. 16 (2014) 20411.

7 D.P. Dubal, J. Suarez-Guevara, D. Tonti, E. Enciso, P. Gomez-Romero, J. Mater. Chem. A 46 (2015) 23483.

8 Z.W. Xian, R.J. Liu, H. Li, S.S. Zhang, Z.H. Yang, W.Q. Zheng, C.H. Chen, H.B. Cao, G.J. Zhang, J. Clust. Sci. 27 (2016) 241.

9 M.T. Pope, Heteropoly, Isopoly Oxometalates, Springer-Verlag: Berlin, Heidelberg, New York, Tokyo, 1983.

10 M.T. Pope, A. Müller, Polyoxometalates: From Platonic Solids to Antiretroviral Activity Kluwer Academic Publications, Dordrecht, 1994.

11 R. Contant, G. Hervé, Rev. Inorg. Chem. 22 (2002) 63. 
12 H. N. Miras, J. Yan, D.-L. Long, L. Cronin, Chem. Soc. Rev. 41 (2012) 7403 .

13 S.S. Wang, G.Y. Yang, Chem. Rev. 115 (2015) 4893.

14 A. Proust, B. Matt, R. Villanneau, G. Guillemot, P. Gouzerh, G. Izzet, Chem. Soc. Rev. 41 (2012) 7605.

15 A. Sartorel, M. Bonchio, S. Campagna, F. Scandola, Chem. Soc. Rev. 42 (2013) 2262.

16 Bassem S. Bassil, U. Z. Kortz, Anorg. Allg. Chem. 636 (2010) 2222.

17 A. Muller, P. Gouzerh, Chem. Soc. Rev. 41 (2012) 7431.

18 H. Lv, Y.V. Geletii, C. Zhao, J.W. Vickers, G. Zhu, Z. Luo, J. Song, T. Lian, D. G. Musaev, C. L. Hill, Chem. Soc. Rev. 41 (2012) 7572.

19 O. Oms, A. Dolbecq, P. Mialane, Chem. Soc. Rev. 41 (2012) 7497.

20 B. Keita, L. Nadjo, J. Mol. Catal. A: Chem. 262 (2007) 190.

21 H.D. Pratt, W.R. Pratt, X.K Fang, N.S. Hudak, T.M. Anderson, Electrochim. Acta 138 (2014) 210.

22 H.G.T. Ly, G. Absillis, R. Janssens, P. Proost, T.N. Parac-Vogt, Angew. Chem. Int. Ed. 54 (2015) 7391.

23 Z.J. Liu, X.L. Wang, C. Qin, Z.M. Zhang, Y.G. Li, W.L. Chen, E.B. Wang, Chem. Rev. 313 (2016) 94.

24 J.J. Stracke, R. G. Finke, ACS Catal. 4 (2014) 909.

25 B. Qin, H. Chen, H. Liang, L. Fu, X. Liu, X. Qiu, S. Liu, R. Song, Z. Tang, J. Am. Chem. Soc. 132 (2010) 2886.

26 Y. Ji, L. Huang, J. Hu, C. Streb, Y. F. Song, Energy Environ. Sci. 8 (2015) 776.

27 M. Yang, B.G. Choi, S.C. Jung, Y.K. Han, Y.S. Huh, S.B. Lee, Adv. Funct. Mater. 24 (2014) 7301.

28 M. Yang, B.G. Choi, Carbon Lett. 18 (2016) 84

29 Y. Chen, M. Han, Y. Tang, J. Bao, S. Li, Y. Lan, Z. Dai, Chem. Commun. 51 (2015) 12377.

30 L.M. Rodriguez-Albelo, G. Rousseau, P. Mialane, J. Marrot, C. Mellot-Draznieks, A. R. Ruiz-Salvador, S. Li, R. Liu, G. Zhang, B. Keita, A. Dolbecq, Dalton Trans. 41 (2012) 9989.

31 L. Lisnard, P. Mialane, A. Dolbecq, J. Marrot, F. Sécheresse, Inorg. Chem. Commun. 6 (2003) 503.

32 W.S. Hummers, R.E. Offeman, J. Am. Chem. Soc. 80 (1985) 1339

33 H. Li, S. Pang, X. Feng, K. Mullen, C. Bubeck, Chem. Commun. 46
(2010) 6243

34 L.L. Feng, M. Fan, Y. Wu, Y. Liu, G.D. Li, H. Chen, W. Chen, D. Wang X. Zou, J. Mater. Chem. A 4 (2016) 6860.

35 A.C. Ferrari, J.C. Meyer, V. Scardaci, C. Casiraghi, M. Lazzeri, F. Mauri, S. Piscanec, D. Jiang, K. S. Novoselov, S. Roth, A. K. Geim, Phys. Rev. Lett. 97 (2006) 187401-1.

36 S. Stankovich, D.A. Dikin, R.D. Piner, K.A. Kohlhaas, A. Kleinhammes, Y. Jia, Y. Wu, S.T. Nguyen, R.S. Ruoff, Carbon 45 (2007) 1558

37 J.S. Qin, D.Y. Du, W. Guan, X.J. Bo, Y.F. Li, L.P. Guo, Z.M. Su, Y.Y. Wang, Y.Q. Lan, H.C. Zhou, J. Am. Chem. Soc. 137 (2015) 7169.

38 D.M. Fernandes, M. Nunes, B.B. Baeza, I.R. Ramos, A.G. Ruiz, C.D. Matos, C. Freire, J. Solid State Electrochem. 21 (2017) 1059.

39 D.M. Fernandes, C. Freire, ChemElectroChem2 (2015) 269.

40 S. Thabet, M. Ayed, B. Ayed, A. Haddad, Journal of Molecular Structure 1075 (2014) 26.

41 Y. Izumi, R. Hasebe, K. Urabe, J. Catal. 84 (1983) 402.

42 M.A. Schwegler, P. Vinke, M. Van der Ejik, H. Van Bekkum, Appl. Catal. A: Gen. 80 (1992) 41.

43 P. Ordejón, E. Artacho, J. Soler, Matter Mater. Phys. 53 (1996) R10441.

44 D. Sánchez-Portal, P. Ordejón, E. Artacho, J.M. Soler, Int. J. Quantum Chem. 65 (1997) 453.

45 J.M. Soler, E. Artacho, J.D. Gale, A. García, J. Junquera, P. Ordejón, D. Sánchez-Portal, J. Phys.: Condens. Matter. 14 (2002) 2745.

46 J.P. Perdew, K. Burke, M. Ernzerhof, Phys. Rev. Lett. 77 (1996) 3865.

47 W. Chaikittisilp, M. Hu, H.J. Wang, H.S. Huang, T. Fujita, K.C.-W. Wu, L.C. Chen, Y. Yamauchi, K. Ariga, Chem. Commun. 48 (2012) 7259.

48 R.R. Salunkhe, J.J. Lin, V. Malgras, S.X. Dou, J.H. Kim, Y. Yamauchi, Nano Energy 11 (2015) 211.

49 R.R. Salunkhe, S.H. Hsu, K.C.W. Wu, Y. Yamauchi, ChemSusChem 7 (2014) 1551

50 R.R. Salunkhe, Y. Kamachi, N.L. Torad, S.M. Hwang, Z.Q. Sun, S.X. Dou, J.H. Kim, Y. Yamauchi, J. Mater. Chem. A 2 (2014) 19848. 